OPEN ACCESS

Edited by: Krisztian Kordas,

University of Oulu, Finland

Reviewed by: Istvan Palinko,

University of Szeged, Hungary Bertil Eliasson,

Umeå University, Sweden

*Correspondence: Xiaohong $\mathrm{Hu}$ huxiaohong07@163.com

Specialty section

This article was submitted to Translational Materials Science, a section of the journal Frontiers in Materials

Received: 21 July 2019 Accepted: 16 April 2020 Published: 27 May 2020

Citation:

Pang J, Kong J, Xu J, Mao X and HuX (2020) Synthesis and Investigation of Macromolecular

Photoswitches. Front. Mater. 7:120. doi: 10.3389/fmats.2020.00120

\section{Synthesis and Investigation of Macromolecular Photoswitches}

\author{
Juan Pang, Jingyang Kong, Jialing Xu, Xincheng Mao and Xiaohong $\mathrm{Hu}^{*}$ \\ School of Material Engineering, Jinling Institute of Technology, Nanjing, China
}

The photoisomerization of azobenzene (AZO) makes it a potential type of photoswitch for the field of chemical engineering. However, the lack of stability and photobleaching characteristics of reversible photoisomerization have restricted further application of AZO as a photoswitch. Therefore, we have designed two polymers containing the AZO domain as macromolecular photoswitches and investigated their switching performance. The hydrophilic monomers hydroxyethyl methylacrylate (HEMA) and N-vinyl-2-pyrrolidone (NVP) were chosen to copolymerize with the AZO monomer to form HEMA-AZO copolymer and HEMA-NVP-AZO terpolymer. The domain content was calculated by integration of the peaks in the ${ }^{1} \mathrm{H}$ NMR spectrum. Real-time UV spectra of polymer solutions upon UV light irradiation confirmed quick and successful trans-to-cis transition for the AZO domain. In reverse, the operable and controllable recovery processes upon white light were also verified by real-time UV spectra. Furthermore, repeated irradiation by UV light and white light for 20 times was used to check their fatigue resistance and recyclability. Importantly, recovery behaviors for the two polymers could be adjusted by solvent property, environment temperature, and light intensity. Higher environment temperature or higher light intensity resulted in shortened recovery time. The influence of solvent was only slightly different for the two polymers. Nanoassemblies, which were formed by HEMA-NVP-AZO terpolymer and poly( $\beta$-cyclodextrin)/poly( $\alpha$-cyclodextrin), exhibited similar controllable switching performance to the pure polymer. In brief, the effectiveness and efficiency of the polymers as photoswitches have been confirmed by results in this work.

Keywords: azobenzene, copolymer, light response property, photoswitch, isomerization

\section{INTRODUCTION}

Organic molecules with reversible isomerization attract increasing interests in numerous fields related to sensors, especially as a kind of switch (Mellerup et al., 2016; Garah et al., 2017; Cardano et al., 2018; Kang et al., 2018; Li et al., 2018; Nacci et al., 2018; Tripathi et al., 2018; Wang et al., 2018; Zhang et al., 2018). Generally, isomerization transition was often excited by a specific stimulus. Among the stimuli, light is commonly used to realize reversible spatiotemporal control. The isomerization of azobenzene upon light makes it a potential optimal photoswitch in 
materials and devices field (Beharry and Woolley, 2011; Ogoshi et al., 2011; Garah et al., 2017; Cardano et al., 2018; Li et al., 2018; Nacci et al., 2018; Pang et al., 2018, 2019; Takeshita and Hara, 2018; Weis and $\mathrm{Wu}, 2018$ ). In natural state, azobenzene exists in trans form due to low intrinsic energy (Crecca and Roitberg, 2006; Corchado et al., 2014). Once the trans isomer is excited by UV light, it converts to cis form immediately. After removing UV light or irradiating by white light, the cis form was recovered to trans form (Beharry and Woolley, 2011; Pang et al., 2018). Unfortunately, the cis form is too unstable to be controllable in the application of small azobenzene molecules as a photoswitch (Beharry and Woolley, 2011; Pang et al., 2014, 2018). Moreover, the transition between trans and cis was easily influenced by environmental factors and type of stimuli. Since no application was in vacuum condition, azobenzene should also satisfy fundamental requests as photoswitches like certain thermostability, fatigue durability, detectability, and non-destructive readability in specific environment. Therefore, designing new azobenzene-based molecules as well as modifying existing azobenzene-based molecules is very necessary and important for their photoswitch applications.

Previously, the photochemistry of azobenzene-containing polymers had been reported and reviewed from the view of chemistry (Sudesh and Neckers, 1989). Recently, different kinds of substitution at opposite and neighbor positions of azobenzenes were designed to improve their properties as photoswitches including our previous research (Fujiwara et al., 2008; Tseng et al., 2012; Li et al., 2013; Angelini et al., 2015; Gon et al., 2019). Furthermore, the response wavenumber could be adjustable by substitute groups according to theoretical calculation. However, these efforts only restricted to the change of substitute groups for azobenzene organic molecules. Furthermore, the problem concerning thermostability of cis form had not been resolved yet.

In one aspect, stability of isomer was related with excited energy and steric effects. Since the transition energy from cis state to excited state is fixed, the steric effect is a good factor to improve thermostability of the cis form. In view of molecule interaction, the neighboring or surrounding molecules have greater influence for central groups. Therefore, copolymerization is considered to be an optimal method for improving thermostability of the cis form in the research.

In other aspect, the problem of unstable switching between cis and trans forms might be ascribed to stacking between molecules, which increase energy barrier to hinder trans to cis transition. In this sense, copolymerization can enlarge distance between azobenzene molecules as well as restrict molecule mobility. Therefore, copolymerization is also hopeful to solve the problem.

Based on the above-mentioned description, copolymers containing azobenzene pendant group was designed and synthesized to solve the problems of azobenzene as photoswitches in the research. Although AZO molecule exhibited obvious response-recovery properties in non-polar environment, it exhibits no light response property in polar solvent especially in water. Therefore, in order to broaden its application environment, two hydrophilic monomers were chosen to copolymerize with AZO monomer to form macromolecular photoswitches. Moreover, factors to influence
TABLE 1 | The feed ratio and structural information for copolymers.

\begin{tabular}{lccccccc}
\hline & \multicolumn{3}{c}{ Feed ratio } & & \multicolumn{2}{c}{ Structural unit proportion } \\
\cline { 2 - 3 } \cline { 6 - 7 } & HEMA & NVP & AZO & & HEMA & NVP & AZO \\
\hline $\begin{array}{l}\text { HEMA-AZO } \\
\text { copolymer (mol\%) }\end{array}$ & 80 & 0 & 20 & & 78 & 0 & 22 \\
$\begin{array}{l}\text { HEMA-NVP-AZO } \\
\text { terpolymer (mol\%) }\end{array}$ & 50 & 30 & 20 & & 54 & 28 & 18 \\
\hline
\end{tabular}

their response and recovery properties were intensively investigated. Finally, a simple and preliminary application as a nanoassembly material was shown in the work.

As a whole, the novelty of the research is to provide available and widely applied photoswitch copolymers. Therefore, systematic light response performances and preliminary application evaluation for synthesized copolymers in different medium environments were investigated in the research.

\section{EXPERIMENTAL}

\section{Material}

Acryloyl chloride, p-aminoazobenzene (m-AZO) was purchased from Aladdin-e Co., Ltd, China. Dichloromethane (DCM), hydroxyethyl methylacrylate (HEMA), $\beta$-cyclodextrin ( $\beta$ CD), $\alpha$-cyclodextrin ( $\alpha$-CD), $N$-vinyl-2-pyrrolidone (NVP), epichlorohydrin, diethyl ether, tetrahydrofuran (THF), dioxane, benzoyl peroxide (BPO), triethylamine (TEA), and dimethylsulfone (DMSO) were obtained from Sinopharm Chemical Reagent Co., Ltd, China. All other reagents and solvents were of analytical grade and used as received from Nanjing Reagent Co.

\section{Synthesis of Polymers}

AZO monomer was synthesized by acylchlorination according to a previous method (Pang et al., 2019). Briefly, m-AZO was reacted with acryloyl chloride in anhydrous DCM at stirred state for $4-5 \mathrm{~h}$. AZO monomer was obtained by purifying procedure and vacuum drying. The detail of processing is shown in the Supporting Information.

HEMA-AZO copolymer and HEMA-NVP-AZO terpolymer were synthesized by radical copolymerization. Briefly, chosen monomers with certain ratio (Table 1) were initiated by $\mathrm{BPO}$ to be polymerized in dioxane solution under anaerobic environment at $70^{\circ} \mathrm{C}$ for $24 \mathrm{~h}$. Polymers were obtained by purifying procedure and freeze drying. Final products were characterized by ${ }^{1} \mathrm{H}$ nuclear magnetic resonance $\left({ }^{1} \mathrm{H}\right.$ NMR, Bruker, AV300). The detail of processing is shown in the Supporting Information.

\section{Response-Recovery Performance for Polymers}

Polymer dilute solution was tracked by UV spectroscopy (Cary 50) for the characterization of its responsive and recovery performance. UV lamp (10 W, 265-420 nm) with lampshade was used as a photo source to induce trans-to-cis transition of AZO 
domain. The UV light density was fixed at $5 \mathrm{~mW} / \mathrm{cm}^{2}$. Reversely, white light and temperature were used to excite cis-to-trans structure recovery. White light irradiation was realized by lightemitting diode (LED) lamp (5 W, 400-750 nm) with lampshade. In addition, light intensity was controlled by irradiation distance and detected by light intensity meter (TA8131). In order to track structural change of molecules, real-time UV spectra as a function of irradiation time and recovery time were recorded. Repeated UV/white light irradiation was applied to demonstrate fatigue resistance of polymers. Furthermore, copolymers in different solvents were checked concerning the influence of polarity for solvent (at least three parallel samples were detected). In addition, the effects of other factors such as temperature and light intensity on recovery performance of copolymers were investigated to clarify structure-property relationship (at least three parallel samples were detected).

\section{Preliminary Application of Polymers as Photoswitches}

$\operatorname{Poly}(\beta-C D) / \operatorname{poly}(\alpha-C D)$ was synthesized by epichlorohydrin crosslinking according to a previous method (Chen et al., 2015). Briefly, $\beta$ - or $\alpha$-CD (2g) was crosslinked by epichlorohydrin $(4 \mathrm{ml})$ into $30 \% \mathrm{NaOH}(10 \mathrm{ml})$ solution at $60^{\circ} \mathrm{C}$. The reaction ended at the point of the solution becoming viscous. The resultant product was obtained by dialyzing and freeze drying after the $\mathrm{pH}$ value of the solution was adjusted to 7 by hydrochloric acid (HCL) dilute solution.

For nanoassemblies, HEMA-NVP-AZO terpolymer was dissolved in DMSO to obtain terpolymer/DMSO solution with $10 \%(W / V)$. Simultaneously, poly $(\beta-C D)$ or poly $(\alpha-C D)$ was dissolved in water to obtain $\operatorname{poly}(\beta-C D)$ or $\operatorname{poly}(\alpha-C D)$ solution with $10 \mathrm{mg} / \mathrm{ml}$. Four hundred microliters terpolymer solution and $4 \mathrm{ml}$ poly $(\beta-C D)$ or poly $(\alpha-C D)$ solution were mixed to obtain nanoparticle, which was further dialyzed to remove DMSO. The obtained nanoassembly was tracked by UV spectroscopy (Cary 50). Different light sources were used to induce trans/cis transition of AZO nanoassembly.

\section{Statistical Analysis}

Data were analyzed using one-way analysis of variance (oneway ANOVA) for differences. Results are reported as means \pm standard deviation. The significant level was set at $p<0.05$; the great significant level was set at $p<0.01$.

\section{RESULTS AND DISCUSSION}

\section{Synthesis of Polymers}

Synthesis of polymer was accomplished by two steps. One was AZO monomer synthesis through acylchlorination according to a previous method (Pang et al., 2019); the other was BPOinitiated radical copolymerization with hydrophilic monomers. Two kinds of copolymer were synthesized for investigation, namely, HEMA-AZO copolymer and HEMA-NVP-AZO terpolymer, which were characterized by ${ }^{1} \mathrm{H}$ NMR spectrum in Figures 1A,B. For HEMA-AZO copolymer (Figure 1A), the details of chemical shift are listed as follows: chemical shifts from 7.4 to $8.5 \mathrm{ppm}$ are attributed to the protons of benzene ring at 1-4 position; chemical shifts from 0.5 to $1.2 \mathrm{ppm}$ are attributed to the protons of methyl at 5 position. Therefore, ${ }^{1} \mathrm{H}$ NMR spectrum confirmed the existence of AZO and HEMA domains of the HEMA-AZO copolymer. Moreover, relative $\mathrm{H}$ content could be calculated by ${ }^{1} \mathrm{H}$ NMR spectrum according to the integration of corresponding peaks since areas of resonance peaks are proportional to number of protons. Furthermore, relative AZO domain content could be calculated to $22 \%$ by the average proton intensity ratio (Table 1 ). In view of feed ratio, the AZO domain ratio was slightly higher than the AZO monomer ratio, which indicated that more AZO structural unit entered the copolymer chain. From the view of radical copolymerization, the AZO monomer might be more active in the polymerization system.

For HEMA-NVP-AZO terpolymer (Figure 1B), besides similar chemical shifts at 1-5 positions to HEMA-AZO copolymer, chemical shifts from 1.2 to $2.3 \mathrm{ppm}$ are attributed to the protons of five-membered ring at 6-8 positions, which confirms the existence of the NVP domain. Analogously, AZO domain content could be calculated to $18 \%$, and NVP domain content could be calculated to $28 \%$ by the average proton intensity ratio (Table 1). Differently, less AZO and NVP unit entered the terpolymer chain, which indicated that HEMA might be more active in this polymerization system.

Comparing the results of HEMA-AZO copolymer with that of HEMA-NVP-AZO terpolymer, it was found that the polymerization activity of the monomer might be different in different polymerization systems. In addition, the HEMA-AZO copolymer and HEMA-NVP-AZO terpolymer could dissolve in DMSO to reach $10 \%(W / V)$ concentration. Although two polymers did not dissolve in water individually, the HEMAAZO copolymer could dissolve in mixed solvent with $90 \%$ water and $10 \%$ DMSO to reach $0.02 \%(W / V)$ concentration, and the HEMA-NVP-AZO terpolymer could dissolved in mixed solvent with $90 \%$ water and $10 \%$ DMSO to reach $0.1 \%$ $(W / V)$ concentration.

\section{Response-Recovery Performance for HEMA-AZO Copolymer}

Photo response of copolymer dilute solution was characterized since polymers might be in undisturbed states in dilute solution. UV light was used as a light source to induce isomerization transition of AZO domain, which was reflected in the UV spectrum from verified theory and previous research (Pang et al., 2018, 2019).

In DMSO dilute solution, HEMA-AZO copolymer response behaviors to UV light and its postresponse behaviors were realtime monitored by UV spectra in Figures 2A,B. In natural state, an obvious peak with maximum absorption value was situated at $360 \mathrm{~nm}$ in Figure 2A, which belonged to $\pi-\pi^{*}$ transition for trans-isomer of the AZO domain according to a previous research (Pang et al., 2019). Upon UV irradiation, the absorbance at $360 \mathrm{~nm}$ decreased from 1.71 to 0.90 with irradiation time until $30 \mathrm{~s}$; at the same time, a small flat absorption peak at $450 \mathrm{~nm}$ became obvious, and its absorbance increased from 0.28 to 0.33 . According to previous theory, the small flat absorption 

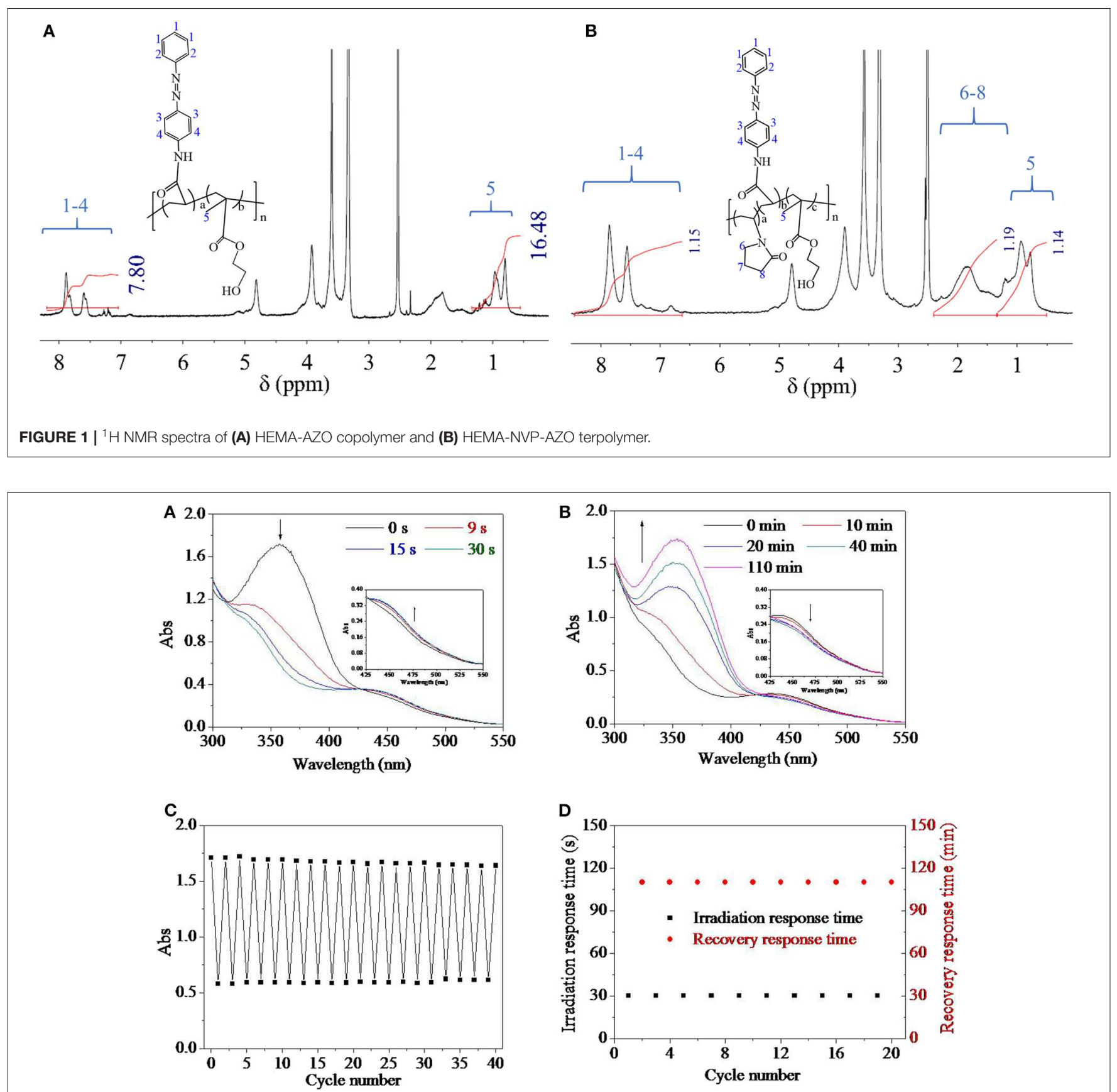

FIGURE 2 | UV spectra of the HEMA-AZO copolymer dimethylsulfone (DMSO) solution as a function of (A) irradiation time and (B) recovery time. (C) Absorbance at $360 \mathrm{~nm}$ of HEMA-AZO copolymer DMSO solution as a function of cycle number. (D) Irradiation response time under UV irradiation and recovery response time under $73 \mu \mathrm{W} / \mathrm{cm}^{2}$ white light as a function of cycle number at $20^{\circ} \mathrm{C}$.

peak was attributed to cis-isomer of the AZO domain. These results indicated that the trans form of the AZO domain could be transferred to the cis form as quick as $30 \mathrm{~s}$ under irradiation by UV. Theoretically, the cis form of the AZO domain is unstable in natural state due to higher intrinsic energy, which can induce recovery of its trans form. For AZO small molecule or AZO-modified polysaccharide derivative, the recovery process could be finished in dark environment at room temperature (Pang et al., 2018, 2019). However, for the HEMAAZO copolymer, the recovery process could not be finished in dark environment at room temperature. Therefore, a white light source was applied to accelerate the recovery process. Upon white light irradiation, absorbance at $360 \mathrm{~nm}$ was gradually recovered to its origin value within $110 \mathrm{~min}$; concurrently, the 


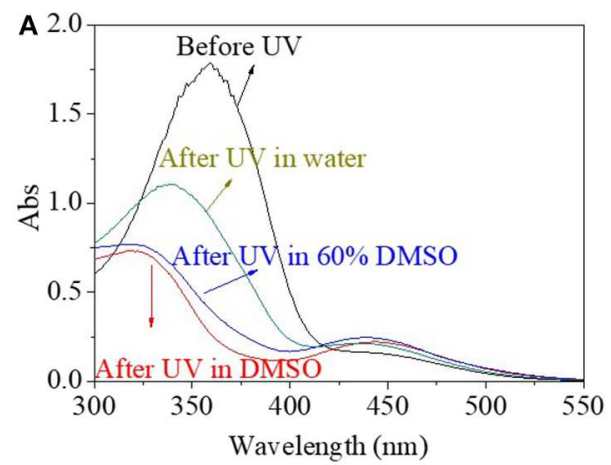

C

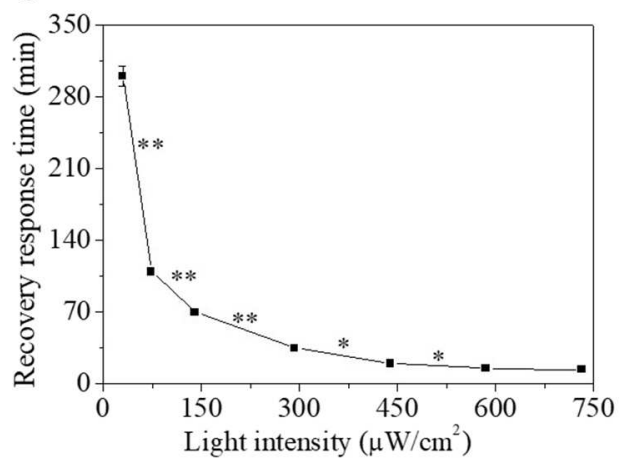

B

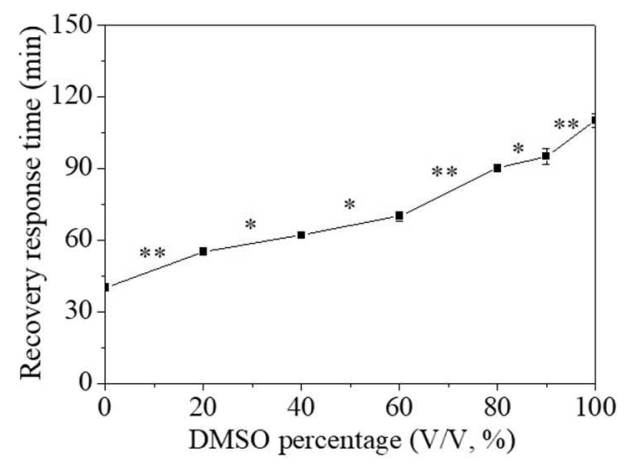

D

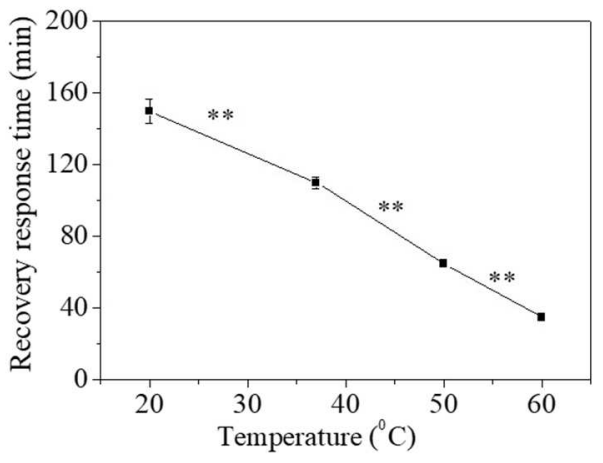

FIGURE 3 | (A) UV spectra of the HEMA-AZO copolymer before and after UV irradiation in dimethylsulfone (DMSO)/ $\mathrm{H}_{2} \mathrm{O}$ solution. (B) The recovery-response time in $\mathrm{DMSO} / \mathrm{H}_{2} \mathrm{O}$ solution as a function of DMSO percentage under $73 \mu \mathrm{W} / \mathrm{cm}^{2}$ white light at $20^{\circ} \mathrm{C}$. The recovery-response time as a function of (C) light intensity at $20^{\circ} \mathrm{C}$ and (D) temperature under $65 \mu \mathrm{W} / \mathrm{cm}^{2}$ white light in DMSO solution. ${ }^{*} p<0.05,{ }^{* \star} p<0.01$. $p$ refers to significance level in the data set.

small flat absorption peak at $450 \mathrm{~nm}$ became unsharp during that period (Figure 2B). These results indicated the recovery of trans structure for the AZO domain. Actually, prolonged recovery time provides a possibility for control factor design as a photoswitch.

Besides response and postresponse behaviors, fatigue resistance and recyclability were evaluated by repeated UV/white light irradiation, which is shown in Figures 2C,D. The maximum absorbance at $360 \mathrm{~nm}$ before UV irradiation or after white light irradiation was stabilized $\sim 1.71$, while the minimum absorbance at $360 \mathrm{~nm}$ after UV irradiation or before white light irradiation was stabilized $\sim 0.6$ regardless of cycle time (Figure 2C). At the same time, regardless of cycle time, irradiation response time was stable at $30 \mathrm{~s}$, and recovery response time was stable at $110 \mathrm{~min}$ (Figure 2D). These results confirmed the excellent switching performance for the HEMA-AZO copolymer without any sign of fatigue, which could be controlled by UV light and white light. Although m-AZO DMSO solution could exhibit detectable trans-tocis transition induced by $\mathrm{UV}$ and visible recovery process, obvious photobleaching phenomenon could not be overcome, which hindered its application as a photoswitch on account of lacking good switching performance (Pang et al., 2018, 2019). From the view of molecular structure, $\pi-\pi$ stacking was a common interaction between conjugated molecules including azobenzenes. Once the stacking occurred in the solution, trans-to-cis transition would become difficult due to larger barrier coming from at least two molecules. Thus, azobenzenes in specific application environment would lose their response property. But differently, when one end of azobenzene was fixed by grafting or polymerization, either the restricted mobility of AZO domain or steric-hindrance effect would prevent stacking between molecules. Therefore, AZO domain onto the polymer chain had good fatigue resistance and exhibited ideal switching performance.

Besides organic solvent, aqueous environment was another frequently used media for their application either as materials or as devices. Therefore, the influence of water was investigated in Figures 3A,B. During the process of research, it was found that the addition of water in the solution had no influence on UV response time of $30 \mathrm{~s}$. However, the UV spectrum of the HEMA-AZO copolymer was influenced by solvent when water percentage in solvent extended $40 \%$ (Figure 3A). Upon UV irradiation in water solution, the maximum absorbance at $360 \mathrm{~nm}$ blue shifted and decreased from 1.71 to 1.01 , and at the same time, the absorbance at $450 \mathrm{~nm}$ increased a little, which was different from that in DMSO solution (Figure 3A). The characteristic of UV spectrum for the HEMA-AZO copolymer in water solution indicated that trans isomer was not converted to cis isomer completely. Furthermore, the recovery-response time shortened significantly with the increase in water percentage in the solution (Figure 3B). Especially, the recovery response time 
in water solution was only $\sim 40 \mathrm{~min}$, which was shorter than that in DMSO solution of $110 \mathrm{~min}$. Incomplete trans-to-cis transition in water solution might result in easier recovery process and shorter recovery response time.

Generally, quick response and controllable recovery are desires for designs of any smart material or device. In order to realize the controllable performance, influence factors should be clarified to reveal the relationship between external stimulus and inner performance. It was found that temperature and light density had substantial effects on the cis-to-trans recovery process, which were shown in Figures 3C,D. The recovery response time declined significantly with increase in white light intensity (Figure 3C). In dark, the cis-to-trans recovery could not be accomplished within 7 days at $20^{\circ} \mathrm{C}$. However, when light intensity increased to $750 \mu \mathrm{W} / \mathrm{cm}^{2}$, the recoveryresponse time shortened to $10 \mathrm{~min}$. Similarly, the recoveryresponse time shortened linearly with increase in temperature with significant difference (Figure 3D). In fact, a premise of either trans-to-cis transition or cis-to-trans transition is to overcome energy barrier from base state to excited state according to existing theory. In the system, UV light source provided higher energy necessary to overcome energy barrier from trans base state to excited state, while white light or high temperature was sufficient for transition from cis base state to excited state. Therefore, higher light intensity or temperature would accelerate cis-to-trans recovery process due to higher input energy. At the same time, trans isomer is more stable than cis isomer in natural state due to its lower energy state.

\section{Response-Recovery Performance for HEMA-NVP-AZO Terpolymer}

Another polymer containing AZO pendant group, the HEMANVP-AZO terpolymer, was also characterized using the abovementioned method.

Real-time response behaviors and its recovery behaviors for the HEMA-NVP-AZO terpolymer in DMSO dilute solution were tracked by UV spectra in Figures 4A,B. UV spectra of the HEMA-NVP-AZO terpolymer in natural state was similar to that of the HEMA-AZO copolymer. After irradiated by UV light, the absorbance at $360 \mathrm{~nm}$ decreased from 1.67 to 0.83 and blue shifted to $345 \mathrm{~nm}$ until $30 \mathrm{~s}$; at the same time, the absorbance of a small flat absorption peak at $450 \mathrm{~nm}$ increased from 0.21 to 0.26 (Figure 4A). After irradiated by white light, peak at $360 \mathrm{~nm}$ was gradually recovered within $115 \mathrm{~min}$; concurrently, the absorbance at $450 \mathrm{~nm}$ decreased a little during that period (Figure 4B). These results indicated that the reversible effective trans-to-cis transition could be excited by UV light and induced to recovery by white light. Furthermore, fatigue resistance and recyclability were evaluated by repeated UV/white light irradiation, which is shown in Figures 4C,D. During the 20 times cycle, the maximum absorbance at $360 \mathrm{~nm}$ before UV irradiation or after white light irradiation was stabilized 1.6-1.7, while the minimum absorbance at $360 \mathrm{~nm}$ after UV irradiation or before white light irradiation was stabilized 0.3-0.5 (Figure 4C). Simultaneously, irradiation response time was stable at $30 \mathrm{~s}$, and recovery response time was stable at $115 \mathrm{~min}$ (Figures 4D). These results also confirmed excellent switching performance for the HEMA-NVP-AZO terpolymer including no sign of fatigue, stable and quick response time, and stable recovery time. The above-mentioned properties for the HEMA-NVPAZO terpolymer were very similar to that for the HEMAAZO copolymer.

Besides organic solvent, the HEMA-NVP-AZO terpolymer in mixed aqueous environment as a function of water percentage is also investigated in Figures 5A,B. The addition of water in the solution had no effect on UV response time, which was $30 \mathrm{~s}$. However, the maximum absorbance at $360 \mathrm{~nm}$ after UV irradiation increased with water percentage when water percentage in solvent extended 90\% (Figure 5A). Furthermore, the recovery response time shortened significantly with the increase in water percentage in the solution (Figure 5B). Especially, the recovery response time in water solution was only $\sim 15 \mathrm{~min}$, which was shorter than that in DMSO solution of 115 min with great significant difference. As discussed above in the part of HEMA-AZO copolymer, trans-to-cis transition for the HEMA-NVP-AZO terpolymer in water could not finish completely, which also influenced recovery-response time. In addition, other influence factors like environment temperature and light density on the cis-to-trans recovery process are investigated in Figures 5C,D. The recoveryresponse time declined great significantly with increase in white light intensity (Figure 5C). In dark, the cis-to-trans recovery could not be accomplished within 7 days at $20^{\circ} \mathrm{C}$. However, when light intensity increased to $750 \mu \mathrm{W} / \mathrm{cm}^{2}$, the recovery-response time shortened to $10 \mathrm{~min}$. Similarly, the recovery-response time shortened with increase in temperature (Figure 5D). As discussed above, higher temperature or light intensity provide extra energy to accelerate cis-to-trans recovery process.

On the whole, the HEMA-NVP-AZO terpolymer possessed similar light-induced switching performance to the HEMAAZO copolymer. However, the influence of solvent on the HEMA-NVP-AZO terpolymer was a little different from that on the HEMA-AZO copolymer. For the HEMA-AZO copolymer, the influence of water on trans-to-cis transition was obvious when water percentage exceeded $40 \%$; for the HEMA-NVP-AZO terpolymer, the influence of water on trans-to-cis transition was obvious when water percentage exceeded $90 \%$. The difference might come from the introduction of NVP domain, which had more hydrophilic characteristic and larger steric effect from its structure characteristic. Therefore, the AZO domain of the HEMA-NVP-AZO terpolymer could have better dispersity in water than that of the HEMA-AZO copolymer. Therefore, in consideration of the adaptation for solvent, the HEMA-NVPAZO terpolymer was chosen for further investigation.

\section{Preliminary Application of HEMA-NVP-AZO Terpolymer}

Due to interactions between AZO domain and hydrophobic cavity of cyclodextrin (CD) (Li et al., 2008; Sogawa et al., 2015), the HEMA-NVP-AZO terpolymer and polyCDs could assemble 

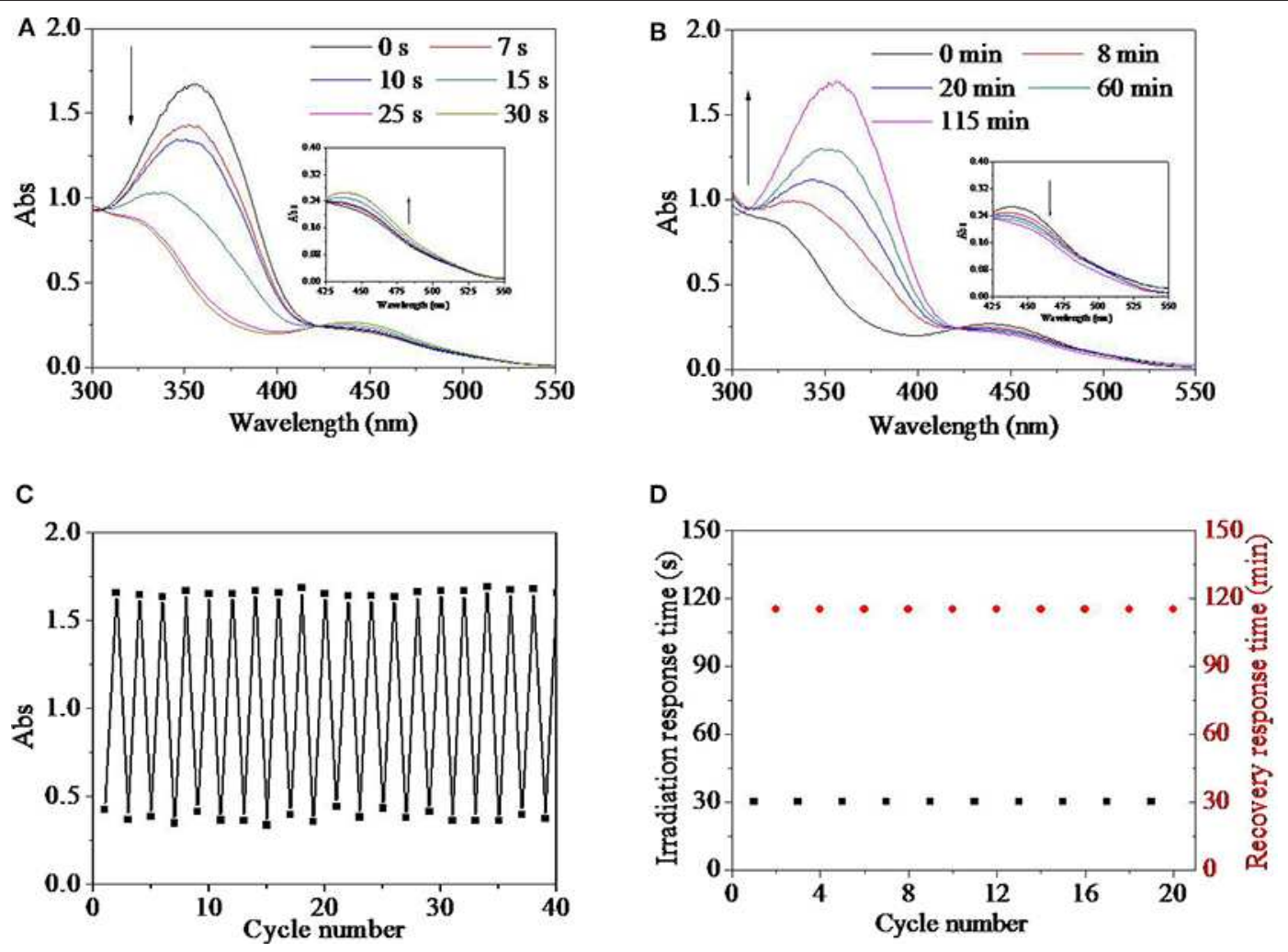

FIGURE 4 | UV spectra of the HEMA-NVP-AZO terpolymer dimethylsulfone (DMSO) solution as a function of (A) irradiation time and (B) recovery time. (C) Absorbance at $360 \mathrm{~nm}$ of the HEMA-NVP-AZO copolymer DMSO solution as a function of cycle number. (D) Irradiation response time under UV irradiation and recovery-response time under $73 \mu \mathrm{W} / \mathrm{cm}^{2}$ white light as a function of cycle number at $20^{\circ} \mathrm{C}$.

into nanoaggregations with effective diameter of several hundred nanometers from DLS results. As a molecule of the HEMANVP-AZO terpolymer, light-controlled switching performance had been proven by the above results. More importantly, the switching performance still existed in materials constructed by the HEMA-NVP-AZO terpolymer. Hence, the light-controlled response-recovery behaviors for terpolymer/poly $(\beta-C D)$ and terpolymer/poly $(\alpha-C D)$ was tracked in Figures $6 \mathbf{A , B}$, respectively. For terpolymer/poly $(\beta-C D)$ in Figure $\mathbf{6 A}$, the absorbance at $360 \mathrm{~nm}$ decreased from 1.73 to 0.89 ; at the same time, the absorbance of a small flat absorption peak at $450 \mathrm{~nm}$ increased from 0.17 to 0.25 on UV irradiation; peaks at 360 and at $450 \mathrm{~nm}$ were gradually recovered to its original value during the period of $110 \mathrm{~min}$ after being induced by white light. Similarly, the response and recovery behaviors for terpolymer/poly $(\alpha-C D)$ nanoassemblies had no obvious difference from that for terpolymer/poly $(\beta-C D)$ nanoassemblies in Figure 6B. Moreover, the fatigue resistance and recyclability of terpolymer/poly-CD nanoassemblies were also similar to that of HEMA-NVP-AZO terpolymer, which was shown in Figure S3 of Supporting Information. These results indicated the effectiveness and efficiency of the HEMA-NVP-AZO terpolymer as a photoswitch either in the form of a single macromolecule or in the form of a material.
Compared with our previous synthesized copolymers containing "AZO pendant groups, two polymers possessed similar photo response behaviors as well as adjustable and controllable recovery properties by factors like light source, light intensity, and temperature (Pang et al., 2019). However, the solvent effect on response and recovery behaviors were not found in previous synthesized HANN copolymers, which might be ascribed to different domain properties for HANN copolymers (Pang et al., 2019).

\section{CONCLUSION}

The HEMA-AZO copolymer and HEMA-NVP-AZO terpolymer were successfully synthesized by radical polymerization. For the HEMA-AZO copolymer, HEMA domain content was calculated to be $78 \%$ and $\mathrm{AZO}$ domain content was calculated to be $22 \%$. For the HEMA-NVP-AZO terpolymer, HEMA domain content was calculated to be $54 \%$, NVP domain content was calculated to be $28 \%$, and AZO domain content was calculated to be $18 \%$. The reversible trans-to-cis transition of both HEMA-AZO copolymer and HEMA-NVP-AZO terpolymer was confirmed by decreasing in absorbance at $360 \mathrm{~nm}$ as well as increasing in absorbance at $450 \mathrm{~nm}$ on real-time UV spectra. UV light source could excite trans-to-cis transition; reversely, white light source could induce cis-to-trans transition. For the HEMA-AZO copolymer, 

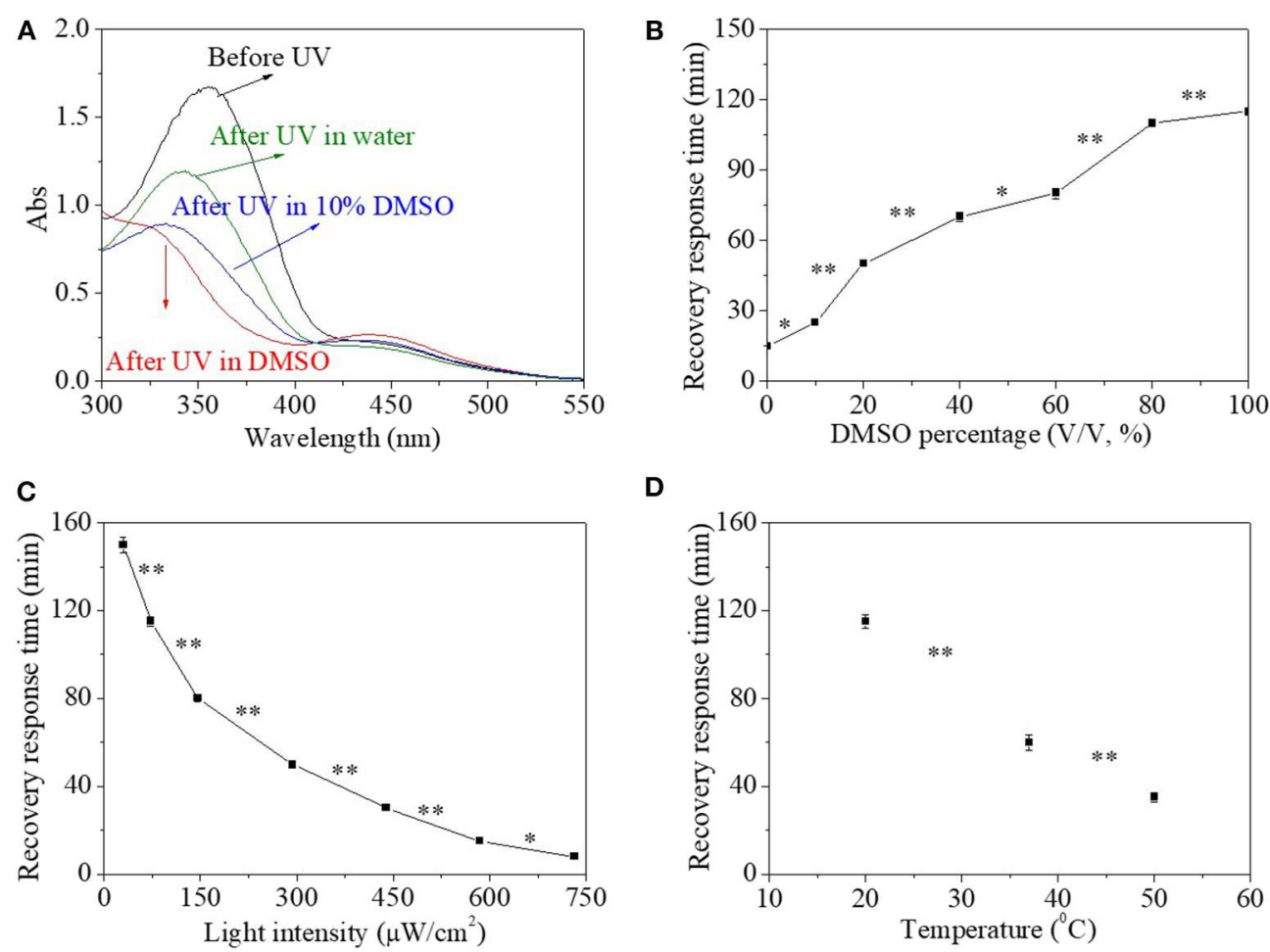

D

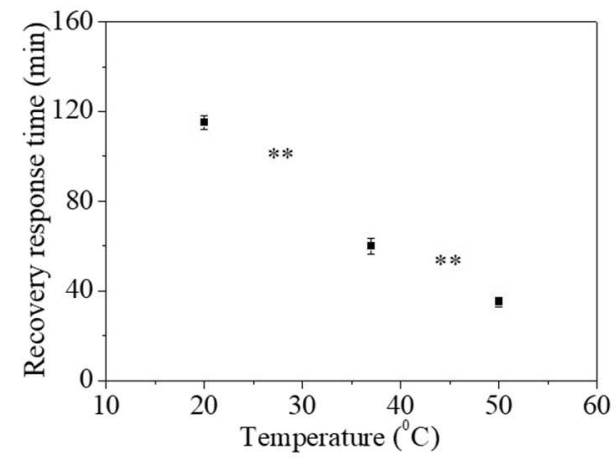

FIGURE 5 | (A) UV spectra of the HEMA-NVP-AZO terpolymer before and after UV irradiation in dimethylsulfone (DMSO)/ $\mathrm{H}_{2} \mathrm{O}$ solution. (B) The recovery-response time in DMSO/ $\mathrm{H}_{2} \mathrm{O}$ solution as a function of DMSO percentage under $73 \mu \mathrm{W} / \mathrm{cm}^{2}$ white light at $20^{\circ} \mathrm{C}$. The recovery-response time as a function of (C) light intensity at $20^{\circ} \mathrm{C}$ and (D) temperature under $73 \mu \mathrm{W} / \mathrm{cm}^{2}$ white light in DMSO solution. ${ }^{*} p<0.05,{ }^{* *} p<0.01 . p$ refers to significance level in the data set.
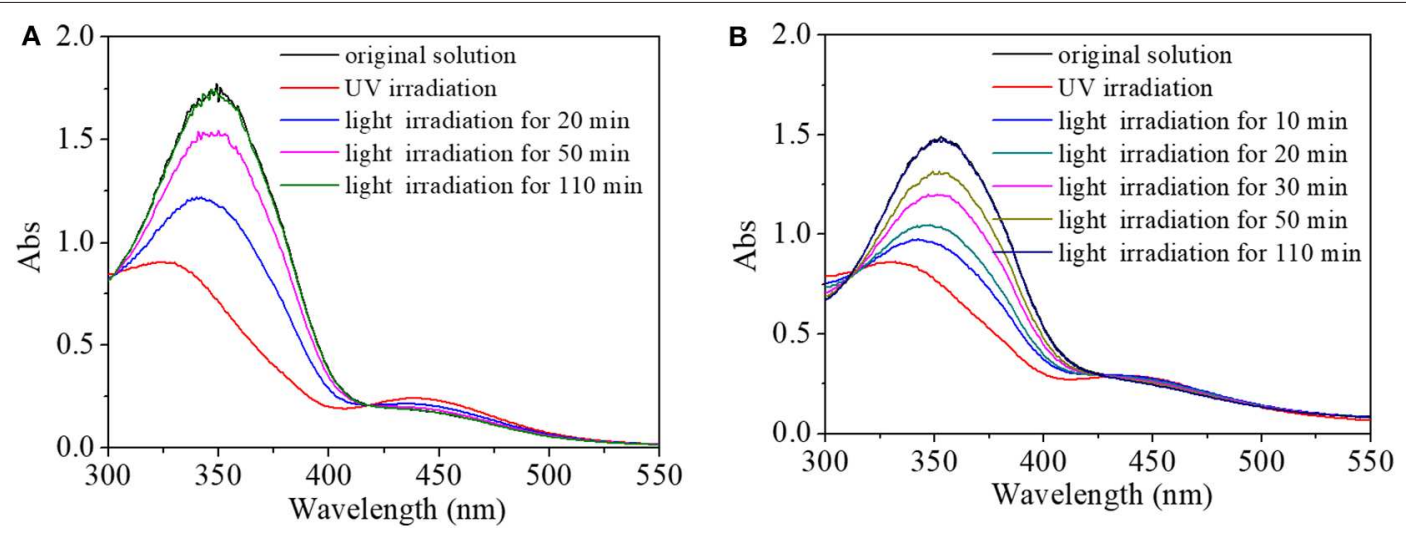

FIGURE 6 | UV spectra of (A) HEMA-NVP-AZO/poly( $\beta-C D)$ and (B) HEMA-NVP-AZO/poly( $\alpha-C D)$ nanoparticle solution response to UV/white light as a function of recovery time under $73 \mu \mathrm{W} / \mathrm{cm}^{2}$ white light at $20^{\circ} \mathrm{C}$.

quick response time of $30 \mathrm{~s}$ and operable recovery time of $110 \mathrm{~min}$ indicated its good performance as a photoswitch. For the HEMA-NVP-AZO terpolymer, quick response time of $30 \mathrm{~s}$ and operable recovery time of $115 \mathrm{~min}$ also confirmed its outstanding properties. In further step, fatigue resistance and recyclability of two polymers were verified by stabilized low absorbance value after UV irradiation and stabilized high absorbance value after recovery during the 20 times double cycle as well as stable response time and recovery time. More importantly, recovery behaviors for two polymers could be adjusted by factors like solvent property, environment temperature, and light intensity. Higher environment temperature or higher light intensity resulted in shortened recovery time for two polymers. However, the influence of solvent was a little 
different. For the HEMA-AZO copolymer, the influence of water on trans-to-cis transition was obvious when water percentage exceeded 40\%; for the HEMA-NVP-AZO terpolymer, the influence of water on trans-to-cis transition was obvious when water percentage exceeded $90 \%$. These characteristics reflected the perfect and controllable switching performance for two polymers in the research. Finally, nanoassemblies formed by the HEMA-NVP-AZO terpolymer and poly $(\beta-\mathrm{CD}) / \operatorname{poly}(\alpha-$ CD) exhibited similar controllable switching performance. In brief, the effectiveness and efficiency of the HEMA-NVP-AZO terpolymer as a photoswitch had been confirmed by results either in the form of a single macromolecule or in the form of a material.

\section{DATA AVAILABILITY STATEMENT}

The datasets generated for this study are available on request to the corresponding author.

\section{REFERENCES}

Angelini, G., Canilho, N., Emo, M., Kingsley, M., and Gasbarri, C. (2015). Role of solvent and effect of substituent on azobenzene isomerization by using roomtemperature ionic liquids as reaction media. J. Org. Chem. 80, 7430-7434. doi: 10.1021/acs.joc.5b00898

Beharry, A. A., and Woolley, G. A. (2011). Azobenzene photoswitches for biomolecules. Chem. Soc. Rev. 40, 4422-4437. doi: 10.1039/c1cs15023e

Cardano, F., Frasconi, M., and Giordani, S. (2018). Photo-responsive graphene and carbon nanotubes to control and tackle biological systems. Front. Chem. 6:102. doi: 10.3389/fchem.2018.00102

Chen, P., Wang, X., Dong, Y., and Hu, X. H. (2015). Development of a layer-bylayer assembled film on hydrogel for ocular drug delivery. Int. J. Polym. Sci. 2015, 1-9. doi: 10.1155/2015/535092

Corchado, J. C., Sanchez, M. L., Fdez Galvan, I., Martin, M. E., Munoz-Losa, A., Barata-Morgado, R., et al. (2014). Theoretical study of solvent effects on the ground and low-lying excited free energy surfaces of a push-pull substituted azobenzene. J. Phys. Chem. B 118, 12518-12530. doi: 10.1021/jp506876v

Crecca, C. R., and Roitberg, A. E. (2006). Theoretical study of the isomerization mechanism of azobenzene and disubstituted azobenzene derivatives. J. Phys. Chem. A 110, 8188-8203. doi: 10.1021/jp057413c

Fujiwara, M., Akiyama, M., Hata, M., Shiokawa, K., and Nomura, R. (2008). Photoinduced acceleration of the effluent rate of developing solvents in azobenzene-tethered silica gel. ACS Nano 2, 1671-1681. doi: 10.1021/nn800290p

Garah, M. E., Borre, E., Ciesielski, A., Dianat, A., Gutierrez, R., Cuniberti, G., et al. (2017). Light-induced contraction/expansion of 1D photoswitchable metallopolymer monitored at the solid-liquid interface. Small 13:1701790. doi: 10.1002/smll.201701790

Gon, M., Wakabayashi, J., Tanaka, K., and Chujo, Y. (2019). Unique substitution effect at 5,5'-positions of fused azobenzene-boron complexes with a $\mathrm{N}=\mathrm{N}$ piconjugated system. Chem. Asian J. 14, 1837-1843. doi: 10.1002/asia.201801659

Kang, W., Zhao, Y., Wang, P., Li, Z., Hou, X., Huang, Z., et al. (2018). Rheological behavior and mechanism of $\mathrm{pH}$-responsive wormlike micelle variations induced by isomers of phthalic acid. Soft Matter 14, 4445-4452. doi: 10.1039/C8SM00467F

Li, L., Wu, R., Guang, S., Su, X., and Xu, H. (2013). The investigation of the hydrogen bond saturation effect during the dipole-dipole induced azobenzene supramolecular self-assembly. Phys. Chem. Chem. Phys. 15, 20753-20763. doi: $10.1039 / \mathrm{c} 3 \mathrm{cp} 52864 \mathrm{~b}$

Li, Y. Y., Jiang, Z. Y., Chen, J. Q., Li, H. L., and Zhang, H. Q. (2008). Environment-friendly determination of low concentration azobenzene

\section{AUTHOR CONTRIBUTIONS}

JP accomplished the manuscript. JK synthesized polymer. JX and $\mathrm{XM}$ characterized materials. XH put an ideal and instruct the whole research.

\section{FUNDING}

This study was financially supported by the Natural Science Foundation of China (21702082), Natural Science Foundation of Jiangsu Province (BK20171113), and Six Talent Peaks project in Jiangsu Province (JY-071).

\section{SUPPLEMENTARY MATERIAL}

The Supplementary Material for this article can be found online at: https://www.frontiersin.org/articles/10.3389/fmats. 2020.00120/full\#supplementary-material

beta-cyclodextrin-modified electrode. Biomed. Environ. Sci. 21, 479-484. doi: 10.1016/S0895-3988(09)60006-4

Li, Z., Yuan, X., Feng, Y., Chen, Y., Zhao, Y., Wang, H., et al. (2018). A reversible conductivity modulation of azobenzene-based ionic liquids in aqueous solutions using UV/vis light. Phys. Chem. Chem. Phys. 20, 12808-12816. doi: 10.1039/C8CP01617H

Mellerup, S. K., Rao, Y. L., Amarne, H., and Wang, S. (2016). Tuning the colors of the dark isomers of photochromic boron compounds with fluoride ions: fourstate color switching. Org. Lett. 18, 4436-4439. doi: 10.1021/acs.orglett.6b02308

Nacci, C., Baroncini, M., Credi, A., and Grill, L. (2018). Reversible photoswitching and isomer-dependent diffusion of single azobenzene tetramers on a metal surface. Angew. Chem. Int. Ed. Engl. 57, 15034-15039. doi: 10.1002/anie.201806536

Ogoshi, T., Yamafuji, D., Aoki, T., and Yamagishi, T. A. (2011). Photoreversible transformation between seconds and hours time-scales: threading of pillar[5] arene onto the azobenzene-end of a viologen derivative. J. Org. Chem. 76, 9497-9503. doi: 10.1021/jo202040p

Pang, J., Gao, Z., Zhang, L., Wang, H., and Hu, X. (2018). Synthesis and characterization of photoresponsive macromolecule for biomedical application. Front. Chem. 6:217. doi: 10.3389/fchem.2018.00217

Pang, J., Gao, Z. Y., Tan, H. P., Mao, X. C., Wang, H. M., and Hu, X. H. (2019). Design, synthesis, investigation and application of a macromolecule photoswitch. Front. Chem. 7:86. doi: 10.3389/fchem.2019.00086

Pang, J., Tian, Z. Q., and Ma, J. (2014). Theoretical design of visible light driven azobenzene-based photo-switching molecules. Chem. Phys. Lett. 613, 110-114. doi: 10.1016/j.cplett.2014.07.048

Sogawa, H., Terada, K., Miyagi, Y., Shiotsuki, M., Inai, Y., Masuda, T., et al. (2015). Photoinduced formation of an azobenzene-based CD-active supramolecular cyclic dimer. Chemistry 21, 6747-6755. doi: 10.1002/chem.201406054

Sudesh, K., and Neckers, G. D. C. (1989). Photochemistry of azobenzenecontaining polymers. Chem. Rev. 89, 1915-1925. doi: 10.1021/cr00098a012

Takeshita, T., and Hara, M. (2018). Photoionization and trans-to-cis isomerization of beta-cyclodextrin-encapsulated azobenzene induced by two-color two-laserpulse excitation. Spectrochim. Acta A Mol. Biomol. Spectrosc. 193, 475-479. doi: 10.1016/j.saa.2017.12.061

Tripathi, S., Bardhan, D., and Chand, D. K. (2018). Multistimuli-responsive hydrolytically stable "smart" mercury(II) coordination polymer. Inorg. Chem. 57, 11369-11381. doi: 10.1021/acs.inorgchem.8b00964

Tseng, C. W., Huang, D. C., and Tao, Y. T. (2012). Electric bistability induced by incorporating self-assembled monolayers/aggregated clusters of azobenzene derivatives in pentacene-based thin-film transistors. ACS Appl. Mater. Interfaces 4, 5483-5491. doi: 10.1021/am3013906 
Wang, Z., Cheng, X., Qin, A., Zhang, H., Sun, J. Z., and Tang, B. Z. (2018). Multiple stimuli responses of stereo-isomers of AIE-active ethynylene-bridged and pyridyl-modified tetraphenylethene. J. Phys. Chem. B 122, 2165-2176. doi: 10.1021/acs.jpcb.7b10929

Weis, P., and $\mathrm{Wu}, \quad$ S. (2018). Light-switchable azobenzene-containing macromolecules: from uv to near infrared. Macromol. Rapid Commun. 39:1700220. doi: 10.1002/marc.201700220

Zhang, Y. P., Chen, L. C., Zhang, Z. Q., Cao, J. J., Tang, C., Liu, J., et al. (2018). Distinguishing diketopyrrolopyrrole isomers in single-molecule junctions via reversible stimuli-responsive quantum interference. J. Am. Chem. Soc. 140, 6531-6535. doi: 10.1021/jacs.8b02825
Conflict of Interest: The authors declare that the research was conducted in the absence of any commercial or financial relationships that could be construed as a potential conflict of interest.

Copyright $\odot 2020$ Pang, Kong, Xu, Mao and Hu. This is an open-access article distributed under the terms of the Creative Commons Attribution License (CC BY). The use, distribution or reproduction in other forums is permitted, provided the original author(s) and the copyright owner(s) are credited and that the original publication in this journal is cited, in accordance with accepted academic practice. No use, distribution or reproduction is permitted which does not comply with these terms. 